\title{
Exposure with response prevention in addition to cognitive behavioural therapy did not decrease bulimic behaviours
}

\author{
Bulik CM, Sullivan PF, Carter FA, et al. The role of exposure with response prevention in the cognitive-behavioural therapy for \\ bulimia nervosa. Psychol Med 1998 May;28:611-23.
}

\section{Questions}

In women with bulimia nervosa, does exposure with response prevention (ERP) added to cognitive behavioural therapy (CBT) improve treatment outcome and prevent relapse? Is ERP to bingeing cues (B-ERP) more effective than ERP to purging cues (P-ERP)?

\section{Design}

Randomised controlled trial with 12 months follow up.

\section{Setting \\ Canterbury, New Zealand.}

\section{Patients}

135 women (mean age 26 y) who had bulimia nervosa according to DSM-III-R criteria. Exclusion criteria included current anorexia nervosa, body mass index $>30 \mathrm{~kg} / \mathrm{m}^{2}$, severe medical illness, complications of bulimia nervosa, and use of psychoactive medication.

\section{Intervention}

After 8 weeks (10 sessions) of CBT, women were allocated to 1 of 3 behavioural treatments for 6 weeks (8 sessions): B-ERP $(\mathrm{n}=37)$, P-ERP $(\mathrm{n}=35)$, or relaxation training $(\mathrm{n}=39)$. For ERP, women were exposed to high risk situations that elicited the urge to binge or purge, but were prevented from doing so for 2.5 hours after the session. Relaxation training included breathing techniques, imagery, and progressive muscle relaxation. Follow up was $86 \%$ for 6 months and $95 \%$ for 12 months.

\section{Main outcome measures}

Core bulimic behaviours (ie, bingeing and purging [clinician rated]) and cue reactivity (physiological ratings and self report scale for urge to binge, urge to purge, and subjective units of distress). Secondary outcome measures were body dissatisfaction, drive for thinness, and bulimia (Eating Disorders Inventory [EDI]); body dissatisfaction (clinician rated); and food restriction.

\begin{abstract}
Main results
Analysis was by intention to treat. The initial CBT was effective; however, no substantial additional benefits from P-ERP compared with B-ERP were seen. After treatment, the groups did not differ for bingeing and purging frequencies. For cue reactivity, B-ERP led to a reduction in peak subjective units of distress compared with relaxation training $(\mathrm{p}=0.001)$. No difference in cue reactivity existed between the relaxation and P-ERP groups. B-ERP led to improvement in reported food restriction (odds ratio [OR] $0.39,95 \%$ CI 0.16 to 1.01 ), clinician rated body dissatisfaction (OR 0.32, CI 0.13 to 0.83), and drive for thinness $(\mathrm{p}=0.01)$ compared with relaxation training. P-ERP led to improvement in bulimic attitudes (EDI) compared with relaxation training $(\mathrm{p}=0.01)$. At 6 months, the differences observed after treatment no longer remained; in addition, body dissatisfaction (EDI) was worse for women in the P-ERP group than in the relaxation group $(\mathrm{p}=0.03)$. At 12 months, the only difference observed was less reported food restriction in the B-ERP group than in the relaxation group $(\mathrm{p}=0.02)$.
\end{abstract}

\section{Conclusions}

In women with bulimia nervosa, cognitive behaviour therapy (CBT) plus exposure with response prevention (ERP) did not reduce core bulimic behaviours or prevent relapse compared with CBT plus relaxation training. ERP to bingeing cues was more effective than ERP to purging cues for decreasing cue reactivity, body dissatisfaction, and food restriction after treatment but not for reducing core bulimic behaviours; at 1 year, the effect remained for food restriction only.

Sources of funding:New Zealand Health Research Council and the New Zealand Lottery Grants Board.

For correspondence:Dr Cynthia M Bulik, Virginia Institute for Psychiatric and Behavioral Genetics, Virginia Commonwealth University, PO Box 980126, Richmond, VA 23298-0126, USA. Fax +1 8048281471

\section{Commentary}

The study by Bulik et al represents the culmination of a large scale, long term project from a well established and respected clinical research team. Other studies on this topic do not use the range of treatment options employed here. In short, the authors have assessed the utility of a novel behavioural component (the rather unfortunately named B-ERP) after CBT for bulimia nervosa. The methodology is clearly reported and allows the reader to follow the twists and turns of such a complex investigation. The authors are particularly clear in dealing with problems of dropouts and in explaining the outcome of some complex statistical analyses.

As regards clinical applications, the easiest conclusion to draw is that neither
B-ERP nor P-ERP have much to offer over and above standard CBT in patients with bulimia. This conclusion supports and extends the existing literature, cited by Bulik et al. However, it would be a mistake to ignore the more important clinical message that underpins this finding-that even relatively brief CBT (10 sessions in 8 weeks) can have a marked effect on bulimic symptoms, and that this benefit is well maintained for a year after treatment. Most manualised versions of clinician delivered CBT used in well controlled trials are substantially longer than this, but have similar outcomes. ${ }^{1}$ In addition, Bulik et al have used fairly broad inclusion criteria relative to some other CBT trials, which means that their findings will apply to a relatively large proportion of the average clinical population. To summarise, clinicians can learn 2 things from this paper. Firstly, they would do well to discover more about this version of CBT (manuals available from Cynthia Bulik). Secondly, there is a clear lesson that exposure with response prevention has little to offer, particularly given its substantial costs.

Glen Waller, DPhil, MClinPsychol University of Southampton Southampton, UK

1 Vitousek KM. The current status of cognitive behavioral models of anorexia nervosa and bulimia nervosa. In: Salkovskis PM, editor. Frontiers of cognitive therapy. New York: Guil-
ford Press, 1996:382-418. 